\title{
Influence of Fractional Electromagnetic Radiation Doses on Biosensor Matrix Component Stability
}

\author{
A. KŁos-WitkowskA \\ Faculty of Mechanical Engineering and Computer Science, Department of Computer Science and Automatics, \\ University of Bielsko-Biała, Willowa 2, 43-309 Bielsko-Biała, Poland \\ (Received July 19, 2017; in final form December 5, 2017)

\begin{abstract}
In the paper influence of electromagnetic field $(200 \mathrm{MHz})$ on biosensor matrix component was shown. Specially designed test bench containing the scanner 3D with the robot was used for electromagnetic field monitoring. The scans of electromagnetic field distribution, before and behind sample were collected. Conformation change of studied protein was monitored by UV spectra. It indicates that fragmentation and aggregation of studied protein might occur with time. The stabilization effect caused by electromagnetic $200 \mathrm{MHz}$ in protein solution was found. The effect was dose dependent (cumulative effect of electromagnetic field) and the differences in the absorption intensity between the control sample and samples after exposition were more visible with time.
\end{abstract}

DOI: 10.12693/APhysPolA.133.101

PACS/topics: biosensor, BSA, UV/Vis, receptor layer, electromagnetic field, stabilization

\section{Introduction}

Influence of electromagnetic field on biological systems has been reported in many investigations [1-3]. Commonly, electromagnetic fields have been used in diagnosing and treating medical therapies [4]. Effects of electromagnetic fields (EMF) application are use in radiotherapy [5], magnetic therapy [6] or electrotherapy (electric current) [7]. Both ionizing and non-ionizing radiation are used.

In all therapies fractional doses are applied to achieve a better, stronger, and more visible effect. Interaction with the electromagnetic field depends on: intensity of the applied field, frequency, duration of exposure, and type of test substance [8].

Electromagnetic wave can be passed through the material, reflected, diffracted, or absorbed. In the case of living organisms (but not only), thermal and non-thermal effects can be observed. In the first case, the energy of the electromagnetic field is changed to heat energy. This effect depends on the frequency and field power influenced on the system. In the second case, the effects are more subtle, usually transient and occur at significantly lower frequencies. Research mechanisms of influence non-ionizing radiation on biological substances are just beginning to develop. It follows that the problem of the impact of non-ionizing electromagnetic fields in combination with chemical agents is important.

On the other hand, biosensors occupy a significant place among the scientific trends. The growing popularity of biosensors is due to the wide range of applications. They are commonly used in environmental protection [9], medicine [10], defense industry; further economic forecasts are very promising.

Based on an economic analysis by Transparency Market Research, the value of the biosensor market in 2018 is estimated at $\$ 18.9$ trillion [11].

Biosensor is a type of sensor based on a biochemicalbiological recognition process consisting of a selective recept/or layer and a transducer layer. In the receptor part, selective uptake of the analyzed molecule (analyte) takes place and this process is possible thanks to the matrix present in this layer. On the surface of the matrix there are receptors that are usually unstable biological molecules such as enzymes, nucleic acids, antigens, cellular organelles, antibodies, whole cells, microorganism. Receptor layer is a very important element of the biosensor, decisive response time and time utility of the equipment [12].

The commonly used substance in biosensor matrix constructions is bovine serum albumin (BSA) [13]. It is used for cross-linking process. BSA is a protein with a molecular weight of $66 \mathrm{kDa}$ consisting of 550 residues. Its crystallographic structure contains nine $\alpha$-helix with cardiac tertiary conformation.

BSA conformational changes caused by varying $\mathrm{pH}$ or temperature were the subject of earlier research $[14,15]$. Reports on the effect of non-ionizing electromagnetic radiation at $200 \mathrm{MHz}$ frequency applied in fractional doses to protein were not found.

Combining these two areas of interest, in the presented work, the following question is going to be answered. How applying an electromagnetic field with a frequency of $200 \mathrm{MHz}$ in fractional doses, on the biosensor matrix component (BSA) can affect its stability. The frequency $200 \mathrm{MHz}$ has been chosen because of existing in literature model, based on magnetization transfer in cross-linked bovine serum albumin solutions [16].

Also $200 \mathrm{MHz}$ was chosen because of the scientific reports about treating the frequency with particular attention. There are reports of influence of the frequency on people (WHO website), and molecular research, where the $200 \mathrm{MHz}$ frequency was used to study sound absorption [17].

\section{Material and methods}

In order to determine the characteristics of applied electromagnetic field to BSA aqueous solution, a special experimental setup was constructed. 
It contains: source of electromagnetic field (wave generator), BSA solutions, EMC-Scanner model RSE321 consisting a $X-Y-Z$ robot, spectrum analyzer with near field probe and a standard computer. Electromagnetic field scans were collected at $200 \mathrm{MHz}$. Scans were made in the $Y Z$ plane before the sample (area $2 \mathrm{~cm} \times 2 \mathrm{~cm}$ ) and behind the sample $(2 \mathrm{~cm} \times 2 \mathrm{~cm})$.

The tests were done on BSA aqueous solutions, basis of scientific reports [18] that such solution provides a better understanding of the physicochemical properties of the test substances. The solutions were exposed to the electromagnetic field applied fractionally in the subsequent experiment days. On the first day of the experiment, a solution of BSA $(2 \mathrm{mg} / \mathrm{ml})$ was made and divided into 4 equal samples.

The first one was the control sample (BSA), the others were designated as:

(RFx1) - sample that was exposed to the $200 \mathrm{MHz}$ electromagnetic field only once in $60 \mathrm{~min}$ on the first day of the experiment.

$(\mathrm{RFx} 2)$ is a sample exposed to the electromagnetic field fractionally twice: on the first day of the experiment (60 $\mathrm{min})$ and on the second day (60 min).

(RFx3) is a sample exposed to electromagnetic fields fractionally triply: on the first day of the experiment (60 min), on the second day (60 min) and on the third day of the experiment (60 min).

Using the double beam U/Vis (Halo DB-20R) spectrophotometer by Dynamica, absorption spectra of the solutions were examined in $(1,2,3,8,10,16)$ days.

\section{Results}

Figure 1 shows scans of the electromagnetic field before the sample (immediately before the source) and behind the sample ( $2 \mathrm{~cm}$ from the source); they were obtained by a specially designed test bench containing the scanner 3D with the robot. The illustration shows a change in electromagnetic field distribution for $200 \mathrm{MHz}$. This change is visible in the image by changing the signal amplitude.

Surprisingly, a slight increase in the amplitude of the signal after passing through the solution can be seen. Contrary to expectations, the electromagnetic field should be weakened with a distance like $1 / r^{2}$, where $r$ is the distance from the source. The response of the biological system to the variable electromagnetic field can be explained by the interference effect of colloids and emulsions in radio frequencies (the Maxwell-Wagner effect), a mechanism of dipolar relaxation in protein solution, and the effect of Grosse [8]. These theories take into account that the responses of biological systems to variable electromagnetic fields depend on dielectric properties: dielectric constant $\varepsilon$ and conductivity $\sigma$, which directly reflect the bound charge and free charge, and which directly have a fundamental contribution to interaction with the field. The fluctuating and selective nature of the response of the substance to the field may also be related to internal interference and to the $\beta$ dispersion [8], which occurs in radio frequencies. According to literature [19, 20], in-
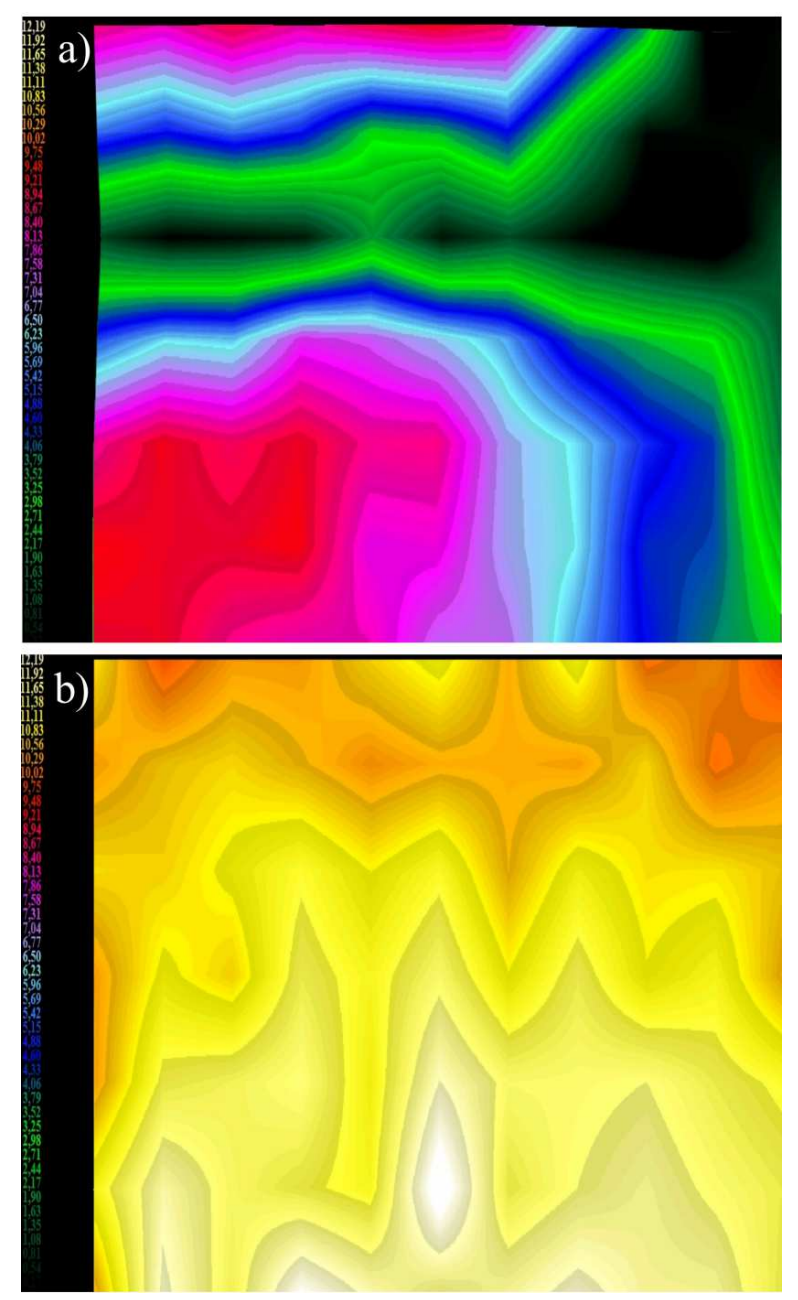

Fig. 1. Electromagnetic field scans for $200 \mathrm{MHz}$ (a) before sample, (b) behind sample. The white color on the spectrum corresponds to the signal amplitude (11.93-12.19 $\mathrm{dB} \mu \mathrm{V})$, yellow $(10.51-11.92 \mathrm{~dB} \mu \mathrm{V})$, orange $(9.76-10.56 \mathrm{~dB} \mu \mathrm{V})$, red $(8.14-9.75 \mathrm{~dB} \mu \mathrm{V})$, pink (6.51-8.13 dB $\mu \mathrm{V})$, blue $(3.80-6.5 \mathrm{~dB} \mu \mathrm{V})$, green $(0.54$ $3.79 \mathrm{~dB} \mu \mathrm{V})$.

ternal reflections lead to the formation of inhomogeneous internal fields and consequent changes in system energy.

It is also worth noting that the research was conducted in a near field where the electromagnetic wave is just beginning to form and the relationship between $E$ and $H$ is complex and difficult to predict.

Figure 2 shows absorption spectra in the range 250 $310 \mathrm{~nm}$ BSA aqueous solutions, made in the following days of the experiment.

The range of spectra was chosen to reflect changes in the $277 \mathrm{~nm}$ peak from the presence of aromatic residuals such as tyrosine (Tyr), phenylalanine (Phe), and tryptophan (Trp). There is an increase in absorption over time. This reflects conformational changes in the secondary structure of the protein, particularly changes in the local environment of the ordered polypeptide chain. This fact may provide decease in BSA stability occurring 
in the protein with time. The changes could reflect in increase of the amount of helix conformation and decrease in beta sheet structure which leads to loosing of the protein skeleton. It also indicates that fragmentation and aggregation of studied protein might occur with time. The protein fragmentation aqueous solution is affected by the local conformation of particular amino-acid.

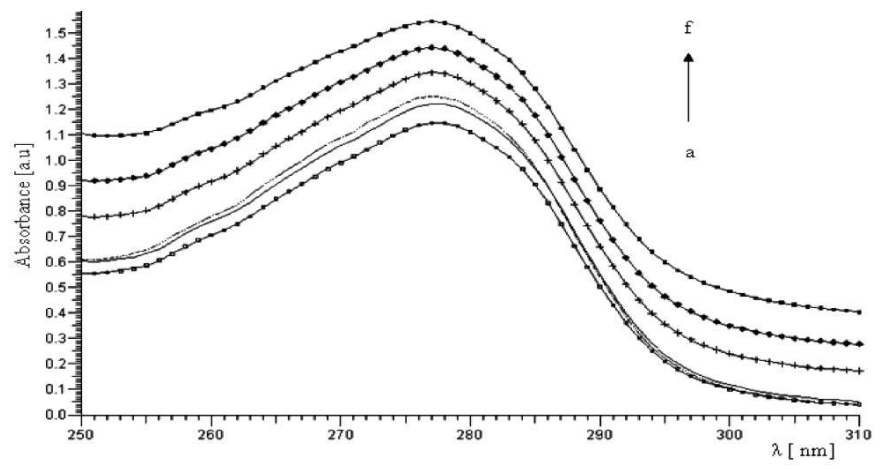

Fig. 2. UV-Vis spectra of aqueous solutions of BSA $(2 \mathrm{mg} / \mathrm{ml})$ without exposure to external agent in the following days: (a) 1 day, (b) 2 days, (c) 3 days, (d) 8 days, (e) 10 days, (f) 16 days.

Over time, denaturation of protein occurs. During this process, a 3-fold structure of the protein is disrupted, resulting in loss of biological activity of the protein. Depending on external factors, the tested component of the biosensor matrix can be crushed in whole or in part only. Takeda et al. [14] have discovered that secondary structural changes occur only after the reduction of disulfide bones. Then the helix content drops from $66 \%$ to $25 \%$, the $\beta$ structure content increases from $3 \%$ to $19 \%$.

The albumin form may change over time and under the influence of external factors such as $\mathrm{pH}$ and temperature. Further conformational changes were labeled as follows: $\mathrm{E}$ - expanded, $\mathrm{F}$ - fast-highly changed state, $\mathrm{N}$ - normal, $55 \% \beta$-helix structure, $\mathrm{B}$ - basic and $\mathrm{A}$ - aged unfolded state. The N-F conformational transition is characterized by a sudden opening of the molecule. The process is very violent and it means $100 \mathrm{~ms}$ and concerns the spreading of the third domain. F form is characterized by much lower solubility and decreased helix structure. $\mathrm{E}$ form is a form of stretched protein, which decomposes into a helix I domain that is joined to the helix II domain. Another known conformation is the conformation $\mathrm{B}$ from which the next isomerization progresses to the ageing forms $\mathrm{A}$.

Figure 3 illustrates the effect of the electromagnetic field put on the biosensor matrix component (BSA) fractionally. The average absorption values with standard deviation from samples exposed to the radio frequency field are shown in the diagram. The values were taken from UV-Vis spectra for samples exposed on RF field only once (on the first day of the experiment, $60 \mathrm{~min}$ ) (RFx1), twice (RFx2) (on the first day of the experiment - $60 \mathrm{~min}$, on the second day $60 \mathrm{~min}$ ) (RFx3) (triple exposure, 1 day - $60 \mathrm{~min}, 2$ days $60 \mathrm{~min}, 3$ days $-60 \mathrm{~min}$ ).
The presented values were collected on the 10th day of the experiment. They show a higher absorption value for the control sample (without field exposure) than for samples treated with external agent. Therefore it is possible to talk about slower conformational changes in radiated samples. By comparing the values derived from the exposure of the external factor in fractional doses, it can be seen that the exposed samples $\mathrm{x} 1$ aged faster than the $\mathrm{x} 2$-treated samples and the longest-stable sample of $\mathrm{x} 3$ exposed.

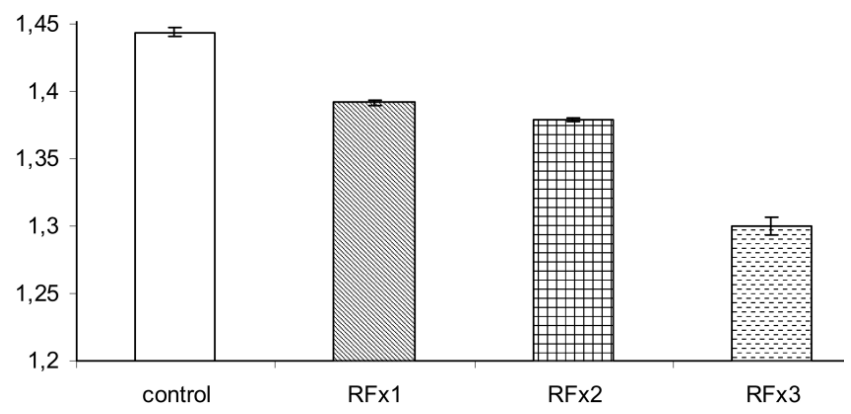

Fig. 3. Cumulative effect of electromagnetic field observed on 10 day of experiment after single (RFx1), double $(\mathrm{RF} \times 2)$, triple $(\mathrm{RF} \times 3)$ radiation.

Therefore we can talk about the cumulative radiofrequency effect [21].

The effect is proportional to cumulative field strength and it is proportional to time average of field strength (because the effect occurs only within field strength window(s) it is seen that $200 \mathrm{MHz}$ is frequency window for aqueous solution of BSA).

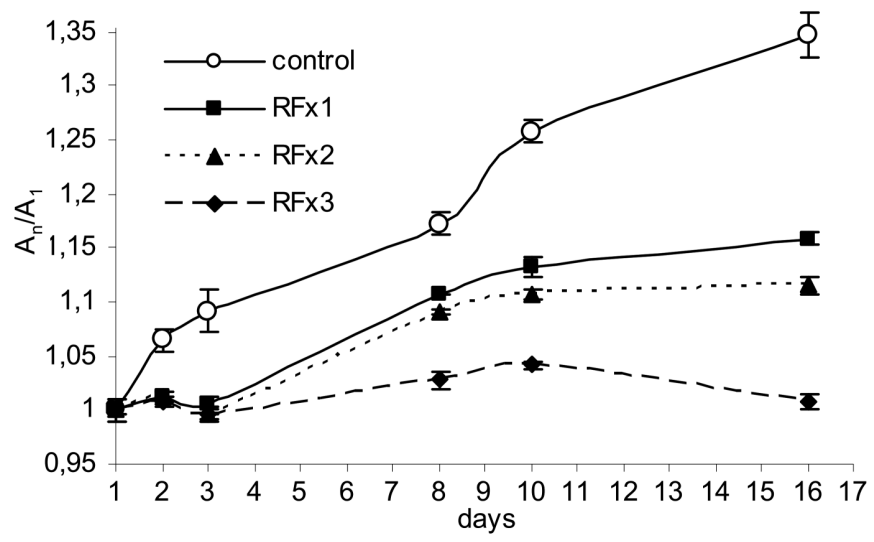

Fig. 4. Relative signal absorption in the following days of experiment.

Changes in absorption intensity over time and under the influence of fractional radiation are shown in Fig. 4. Relative absorption was calculated by $A_{n} / A_{1}$, where $A_{n}$ was absorption in the following days, $A_{1}$ - absorption in the first day of experiment.

Slower increase in absorption with time for samples exposed on electromagnetic field was shown. It can easily be seen that the difference in the absorption intensity between the control samples and the irradiated samples 
are more visible with time. Studies have shown that radiation of $200 \mathrm{MHz}$ frequency slows denaturing process occurrence with time in the test protein. It is therefore possible to suggest the stabilizing effect of a $200 \mathrm{MHz}$ electromagnetic field on a biosensor matrix component.

Slower processes (in proteins subjected to electromagnetic field) may be caused by a weak electrical field (compared to control samples) over a longer period of time (compared to control samples). The electric field resulted from interaction of the solvent with the dissolved molecules. In addition, when comparing the protein solution radiated once and repeatedly, it appears that changes occurring (after exposure) over time appear to be less than for one-day samples. It is also possible to see here the effect of the electromagnetic field accumulation described in literature [22, 23].

\section{Conclusion}

In summary, the studies showed an increase in absorption associated with conformational changes in BSA with time.

The impact of the $200 \mathrm{MHz}$ electromagnetic field on protein stability was observed. Therefore it could be said that exposure to a factor slows the conformational changes that occur in the protein over time and affects its structural modification.

Comparing the effect of influence RF on protein in fractions (single, double, triple), a greater effect on the stabilization of BSA after multiple factor radiation than once was observed.

The field cumulative effect of $200 \mathrm{MHz}$ in aqueous albumin solutions has been found.

Referring the obtained results for biosensors research, it can be said that the $200 \mathrm{MHz}$ electromagnetic field has a stabilizing the receptor layer component (BSA) and this effect is stronger after several fractional doses application.

\section{References}

[1] B. Lewczuk, G. Redlarski, A. Zak, N. Ziółkowska, B. Przybylska-Gornowicz, M. Krawczuk, Biomed. Res. Int. 2014, 169459 (2014).

[2] P. Raif, A. Kłos-Witkowska, M. Mahmud, R. Suchanek, A. Hussain, in: IEEE Symp. on Computational Intelligence in Healthcare and $e$ Health (CICARE) 2013.
[3] V. Pohrebennyk, R. Politylo, V. Yakovleva, I. Salamon, J. Acta Facultatis Studiorum Humanitatis et Naturae Universitata Presoviensis, Natural Sciences, Slovak Republic, Prešov XLIII, 135 (2016).

[4] M. Vadalà, A. Vallelunga, L. Palmieri, B. Palmieri, J. Cesar Morales-Medina, T. Iannitti, Behav. Brain Funct. 11, 26 (2015).

[5] F. Artacho-Cordón, M. Salinas-Asensio, I. Calvente, S. Ríos-Arrabal, J. León, E. Román-Marinetto, N. Olea, Int. J. Mol. Sci. 14, 14974 (2013).

[6] J. Fang, M. Zhou, M. Yang, C. Zhu, L. He, Cochrane Database Syst. Rev. 31, CD008554 (2013).

[7] J. Łazowski, Podstawy fizykoterapii, Wydawnictwo AWI, Wrocław 2009 (in Polish).

[8] C. Polk, E. Postow, Handbook of Biological Effects of Electromagnetic Fields, Vol. 2, 3rd ed., CRC Press, 1995.

[9] A. Kłos-Witkowska, Pol. J. Environm. Stud. 24, 19 (2015).

[10] A. Kłos-Witkowska, Acta Biochim. Pol. 63, 215 (2016).

[11] Transparency Market Research, Biosensors Market (Electrochemical, Optical, Piezoelectric \&5 Thermistor) - Global Industry Analysis, Size, Share, Growth, Trends and Forecast, 2012-2018, 2013-06-27.

[12] A. Kłos-Witkowska, Pomiary Automatyka Kontrola 12, 3 (2014), (in Polish).

[13] A.T. Buddanavar, S.T. Nandibewoor, J. Pharmaceut. Anal. 7, 148 (2017).

[14] K. Takeda, A. Wada, K. Yamamoto, Y. Moriyama, K. Aoki, J. Protein Chem. 8, 653 (1989).

[15] M.A. Masuelli, Adv. Phys. Chem. 13, 360239 (2013).

[16] F. Fisher, J. Acoust. Soc. Am. 111, 2399 (2002).

[17] Electromagnetic fields and public health: radars and human health, WHO, 2017.

[18] A. Michnik, K. Michalik, Z. Drzazga, J. Photochem. Photobiol. B Biol. 90, 170 (2008).

[19] S. Alekseev, M. Zaskin, Bioelectromagnetics 21, 264 (2000).

[20] S. Alekseev, M. Zaskin, Bioelectromagnetics 22, 288 (2000).

[21] D.O. Carpenter, S. Ayrapetyan, Biological Effects of Electric and Magnetic Fields. Benefical and Harmful Effects, Academic Press, 1994.

[22] M. Atef, M. Abd El-Baset, Int. J. Biol. Macromol. 17, 105 (1995).

[23] S. Baranski, Am. J. Physiol. Med. 51, 182 (1972). 